\title{
ON CERTAIN RULED HYPERSURFACES OF ORDER $k-1$ IN $r$-SPACE
}

BY B. C. WONG

This note proposes to prove the following theorem.

THEOREM 1. The locus of the lines incident with $k$ given linear mutually skew sub-spaces of dimensions $t_{1}, t_{2}, \cdots, t_{k}$, respectively, in $r$-space such that

$$
k-2 \leqq t_{1} \leqq t_{2} \leqq \cdots \leqq t_{k} \leqq r-2,
$$

and

$$
t_{1}+t_{2}+\cdots+t_{k}=r k-r-k
$$

is a hypersurface of order $k-1$.

As an immediate consequence of this theorem we have the following.

THEOREM 2. The order of the ruled hypersurface whose generators have simple incidence with each of $k$ varieties of orders $n_{1}, n_{2}, \cdots, n_{k}$ and dimensions $t_{1}, t_{2}, \cdots, t_{k}$ respectively in $r$-space where $r, k$, $t_{1}$, etc., satisfy (1) and (2) is $(k-1) n_{1} n_{2} \cdots n_{k}$.

The case for which $k=r$ and $t_{1}=t_{2}=\cdots=t_{k}=r-2$ is known.* Thus, if $k=r=3$ and $t_{1}=t_{2}=t_{3}=1$, we have a ruled surface of order $2 n_{1} n_{2} n_{3}$ whose rulings meet three curves of orders $n_{1}, n_{2}, n_{3}$ respectively in 3 -space. $\dagger$ The theorems stated above include all the cases for which $2 \leqq k \leqq r$. Thus, if $k=4$, the locus of the lines incident with four sub-spaces of appropriate dimensions in $r$-space is always a cubic hypersurface. We need prove Theorem 1 only, since Theorem 2 follows from it immediately.

To avoid too complicated a system of notations we use $\{x\}$ in place of $S_{x}$ to designate an $x$-space. Then the given $k$ sub-

* Segre, Encycklopädie der Mathematischen Wissenschaften, vol. III C7, pp. 815, 832. Also B. C. Wong, On a certain system of $r-2$ lines in $r$-space, and $O n$ the loci of the lines incident with $k(r-2)$-spaces in $S_{r}$, this Bulletin, vol. 34 (1928), pp. 553-554, and pp. 715-717.

$\dagger$ Salmon, Analytic Geometry of Three Dimensions, 5th ed., vol. II, $\$ 467$. 
spaces of dimensions $t_{1}, t_{2}, \cdots, t_{k}$ will be denoted by $\left\{t_{1}\right\}$, $\left\{t_{2}\right\}, \cdots,\left\{t_{k}\right\}$ respectively.

Let $N$ be the order of the hypersurface $V_{r-1}^{N}$ in $r$-space satisfying the conditions imposed. We are to prove

$$
N=k-1 \text {. }
$$

A $\left(t_{1}+1\right)$-space $\left\{t_{1}+1\right\}$ passing through $\left\{t_{1}\right\}$ meets the other $k-1$ sub-spaces $\left\{t_{i}\right\}$ in the spaces $\left\{t_{i}{ }^{\prime}\right\}$ where $t_{i}{ }^{\prime}=t_{1}+t_{i}+1-r$ $[i=2,3, \cdots, k]$. The lines which meet $\left\{t_{i}^{\prime}\right\}$ form a $V_{t_{1}}^{N-1}$ in $\left\{t_{1}+1\right\}$ which together with $\left\{t_{1}\right\}$ constitutes the intersection of $\left\{t_{1}+1\right\}$ and $V_{r-1}^{N}$. Now a $\left(t_{2}{ }^{\prime}+1\right)$-space $\left\{t_{2}{ }^{\prime}+1\right\}\left[t_{2}{ }^{\prime}\right.$ $\left.=t_{1}+t_{2}+1-r\right]$ passing through $\left\{t_{2}{ }^{\prime}\right\}$ and belonging to $\left\{t_{1}+1\right\}$ meets the other $k-2 t_{i}{ }^{\prime}$-spaces of $\left\{t_{1}+1\right\}$ in the spaces $\left\{t_{i}{ }^{\prime \prime}\right\}$ where $t_{i}^{\prime \prime}=t_{1}+t_{2}+t_{i}+2-2 r[i=3,4, \cdots, k]$. This $\left\{t_{2}^{\prime}+1\right\}$ meets $V_{t_{1}}^{N-1}$ in $\left\{t_{2}{ }^{\prime}\right\}$ and a $V_{t_{2}^{\prime}}^{N-2}$ which is the locus of the lines incident with the spaces $\left\{t_{i}^{\prime \prime}\right\}$.

We have so far gone through two steps in the reasoning. Continuing this process, we soon arrive at the $j$ th step that a $\left(t_{j}^{(j-1)}+1\right)$-space $\left\{t_{j}^{(j-1)}+1\right\}$ passing through $\left\{t_{j}^{(j-1)}\right\}$ where $t_{j}^{(j-1)}=t_{1}+t_{2}+\cdots+t_{j}+(j-1)(1-r)$ and belonging to $\left\{t_{j-1}^{(j-1)}\right\}$ meets the other $k-j\left(t_{i}^{(j-1)}\right)$-spaces of $\left\{t_{j-1}^{(j-2)}\right\}$ in the spaces $\left\{t_{i}^{(j)}\right\}$ where $t_{i}^{(j)}=t_{1}+t_{2}+\cdots+t_{j}+t_{i}+j(1-r) \quad[i=j+1$, $j+2, \cdots, k]$. The locus of the lines meeting $\left\{t_{i}^{(j)}\right\}$ is a $V_{m}^{N-j}$ where $m=t_{j}^{(j-1)}$ in $\{m+1\} \equiv\left\{t_{j}^{(j-1)}+1\right\}$. This $V_{m}^{N-j}$ and $\{m\} \equiv\left\{t_{j}^{(j-1)}\right\}$ form the complete intersection of $\{m+1\}$ with $V_{m^{\prime}}^{N-j+1}$ where $m^{\prime}=t_{j-1}^{(j-2)}$ which is supposed to have been obtained in the $(j-1)$ th step.

Now let $j=k-2$. We have a $\left\{t_{k-2}^{(k-3)}+1\right\}$ or $\{T\}\left[T=t_{k-2}^{(k-3)}+1\right.$ $=2 r-2-t_{k}-t_{k-1}$ on account of (2)] passing through $\{T-1\}$ and belonging to $\left\{t_{k-3}^{(k-4)}\right\}$ which meets the other two spaces $\left\{t_{k-1}^{(k-1)}\right\}$ and $\left\{t_{k}^{(k-3)}\right\}$ of $\left\{t_{k-3}^{(k-4)}\right\}$ in the spaces $\left\{T^{\prime}\right\}$ and $\left\{T^{\prime \prime}\right\}$ respectively where

$$
T^{\prime}=t_{k-1}^{(k-2)}=r-2-t_{k}
$$

and

$$
T^{\prime \prime}=t_{k}^{(k-2)}=r-2-t_{k-1} .
$$

The locus of lines incident with $\left\{T^{\prime}\right\}$ and $\left\{T^{\prime \prime}\right\}$ is a $V_{T-1}^{N-k+2}$ in $\{T\}$. Since the locus of lines meeting two sub-spaces $\left\{n_{1}\right\}$, 
$\left\{n_{2}\right\}$ of $\{n\}$ such that $n=n_{1}+n_{2}+2$ is an $\{n-1\}$, the $V_{T-1}^{N-k+2}$ is a $\{T-1\}$ in $\{T\}$, for $T=T^{\prime}+T^{\prime \prime}+2$. Hence, we have

or

$$
N-k+2=1 \text {, }
$$

$$
N=k-1 \text {, }
$$

which was to be proved.

It is about impossible to state generally any proposition concerning the properties of the hypersurfaces of this type. The properties of some of them can be obtained readily.

The University of California

\title{
A NECESSARY AND SUFFICIENT CONDITION FOR THE NON-EQUIVALENCE OF ANY TWO RATIONAL GENERALIZED QUATERNION DIVISION ALGEBRAS*
}

\author{
BY A. A. ALBERT
}

Two algebras $\mathfrak{A}$ and $\mathfrak{B}$ over the same field $F$ are called equivalent (or simply isomorphic) if it is possible to establish between their quantities a (1-1) correspondence such that if any quantities $x$ and $y$ of $\mathfrak{A}$ correspond to $X$ and $Y$ of $\mathfrak{B}$, then $x+y, x y$ and $\alpha x$ correspond to $X+Y, X Y, \alpha X$ respectively for every $\alpha$ of $F$. We shall consider two generalized quaternion division algebras $\nmid \mathfrak{A}$ and $\mathfrak{B}$ over the field of all rational numbers, $R$. Let $\mathfrak{A}$ be given by

(1) $\mathfrak{A}=\left(e, i_{1}, j_{1}, i_{1} j_{1}\right), i_{1}^{2}=\rho_{1} e, j_{1}^{2}=\sigma_{1} e, j_{1} i_{1}=-i_{1} j_{1}$,

where $e$ is the modulus of $\mathfrak{A}$, and $\rho_{1}$ and $\sigma_{1}$ are in $R$. Without loss of generality $\rho_{1}$ and $\sigma_{1}$ may be taken to be each products of distinct rational prime integers.

Similarly let

(2) $\mathfrak{B}=\left(E, I_{1}, J_{1}, I_{1} J_{1}\right), I_{1}^{2}=\rho_{2} E, J_{2}^{2}=\sigma_{2} E, J_{1} I_{1}=-I_{1} J_{1}$,

* Presented to the Society, February 22, 1930.

$\uparrow$ For the definition and properties of these algebras see L. E. Dickson, Algebren und ihre Zahlentheorie, pp. 46-49. 